\title{
7. ALGUNAS NOVEDADES COROLÓGICAS RELEVANTES EN CIPERÁCEAS IBÉRICAS Y BALEÁRICAS
}

\author{
Modesto LUCEÑO y Pedro JIMÉNEZ-MEJÍAS
}

\section{Some new chorological records on Iberian and Balearic Cyperaceae}

Palabras clave. Cyperaceae, corología, Flora Iberica, España, Portugal, Islas Baleares.

Key words. Cyperaceae, chorology, Flora Iberica, Spain, Portugal, Balearic Islands.

A raíz de algunas campañas de recolección y de la revisión de los materiales de herbarios españoles y portugueses de la familia Cyperaceae, con motivo de la redacción de los manuscritos para Flora Iberica, hemos detectado localidades nuevas de algunas ciperáceas que estimamos relevantes desde el punto de vista corológico. Estas son las siguientes:

\section{Carex oedipostyla Duval-Jouvé}

PORTUGAL, ALGARVE: Entre Barranco do Velho y el pico Bispo; alcornocal atravesado por un arroyo, 29-IV-2005; P. Jiménez Mejías, M. Luceño, A.M. Escudero \& J.F. Bautista. UPOS s/n.

Primera cita en el S de Portugal de una especie poco común en la Península; las localidades más próximas se encuentran en el $\mathrm{C}$ de Portugal (Sintra) y en el Parque Natural de Los Alcornocales $(\mathrm{Ca})$, de donde fue recientemente citada (Luceño \& Marín, Ruizia 14. 2001). Contra lo que se ha supuesto (Maire, 1957), es particularmente abundante en el $\mathrm{N}$ de África. Esta planta ha sido frecuentemente confundida con Carex depressa Link, con la que cohabita frecuentemente, y de la que se distingue bien por sus espigas andróginas y su evidente estilopodio, como fielmente refleja su

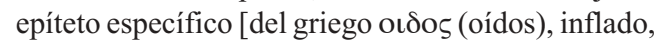

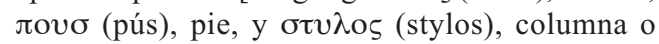
estilo].

\section{Carex pseudocyperus L.}

CÁCERES: Talayuela; río Tiétar, arenas temporalmente inundadas. $275 \mathrm{~m}, 1-\mathrm{X}-2005 ; M$. Luceño, P. Jiménez Mejías, M. Guzmán \& P. Vargas. UPOS718.

Primera cita para Extremadura $(\mathrm{Cc})$ de una especie conocida hasta el momento del $\mathrm{N}$ de España, puntos aislados del CW de Portugal y Doñana (Luceño, Ruizia 14. 1994).

Eleocharis flavescens (Poir.) Urban

PALMA DE MALLORCA. ISLAS

BALEARES [IBIZA]. San Miguel; Fuente d'es

Tur, im Torrentabschnitt östl, 10-XI-1971; $H$. Kuhbier. SEV23659.

Primera cita española de esta planta originaria de América, aunque ya se conocía su introducción en zonas litorales del C de Portugal y el N de Italia (Walters, Fl. Eur. 5: 282. 1980).

Eleocharis mamillata (H. Lindb. fil.) H. Lindb. fil. subsp. austriaca (Hayek) Strandh.

ASTURIAS: Lago Ercina, Covadonga, 16VIII-1951; Emilio Guinea. MA 164579. LÉRIDA: Valle de Arán, puerto de la Bonaigua, bordes de un pequeño lago casi colmatado, $2012 \mathrm{~m}, 16$-VIII2005; M. Luceño, P. Jiménez Mejías, M. Escudero \& S. Martín. UPOS 1400. TERUEL: La Fresneda, hacia Valderrobres, $500 \mathrm{~m}, 0^{\circ} 1$ ' $58^{\prime \prime}$ 'E $40^{\circ} 52^{\prime} 58^{\prime \prime}$ N, 28-V-1989; G. Mateo.MA475216 (mezclada con E. palustris). Idem, Bueña, márgenes de un lagunazo, 1200 m, 22-VI-1988; G. Mateo. MA 465990.

Esta especie eurosiberiana apenas alcanza la península Ibérica, de donde únicamente se tenía constancia de dos localidades pirenaicas: Irati en Navarra y Ordesa en Huesca (Guzmán Otano \& Goñi Martínez, Atlas Libro Rojo Fl. España: 238239. 2004). La población leridana consta de un número elevado de individuos, y en la turolense se pueden observar ejemplares transicionales hacia la muy común E. palustris subsp. palustris. La especie ha sido incluida en el Atlas y Libro Rojo de la Flora Vascular Amenazada de España 
(Bañares \& al., 2003) con la categoría "En peligro crítico" (VV. AA. Conserv. Veg. 6. 2000).

\section{Rhynchospora modesti-lucennoi Castrov.}

PORTUGAL: Bajo Alentejo, Alcácer do Sal; laguna de agua dulce a poca distancia de Montevil, márgenes turbosos, $11 \mathrm{~m}, 8^{\circ} 40^{\prime} 5^{\prime}$ " W 38 23' 43" N, 30-IV-2005; P. Jiménez Mejías, M. Luceño, M. Escudero \& J.F. Bautista. UPOS 1394.

Endemismo ibero-norteafricano en franca regresión debido a la alteración de su hábitat. Hasta ahora se había encontrado en el Parque Natural de Los Alcornocales (Cádiz), en las cercanías de Doñana, en la provincia de Pontevedra -de donde parece haberse extinguido (Ortiz Herrera et al., 2004)- y en puntos aislados de las provincias portuguesas de Estremadura y Beira Litoral. En España se le ha asignado la categoría de "En peligro crítico" (VV.AA., Conserv. Veg. 6. 2000).

Schoenoplectus erectus (Poir.) Palla ex J. Raynal PORTUGAL, BEIRA LITORAL: Figueira da Foz; Quiaios, Lagoa das Braças, 26-X-1990; J. C. Costa, M. Lousã \& R. Molina. LISI s/n.

Primera cita para el país vecino de esta pequeña ciperácea, que ya se conocía del litoral onubense [cf. Gallego Fl. Andalucía Occ. 3: 233, 1987, sub Scirpus uninodis (Delile) Boiss.]. Las plantas de esta localidad lusitana habían sido previamente confundidas con la afín Schoenoplectus supinus (L.) Palla. La especie se encuentra ampliamente distribuida por buena parte del Globo (África, SE de Asia, USA, México, Argentina y Paraguay), y las localidades más próximas a la península Ibérica se hallan en Libia. No tenemos certeza del carácter autóctono de dichas poblaciones; el hecho de que no se conozcan citas antiguas y la distribución antes indicada, nos han inclinado a considerarlas provisionalmente como naturalizadas.

\section{Schoenoplectus pungens (Vahl) Palla}

HUELVA: Puerto de la Laja, El Granado, 15-VII-2001; E. Sánchez Gullón \& J. Macías. MA 695315.

Primera referencia andaluza de este elemento anfiatlántico. La población que ahora citamos es la más meridional del continente europeo. En España se le ha asignado la categoría "En Peligro" (VV.AA., loc. cit. 2000).
Schoenoplectus triqueter (L.) Palla

HUELVA: Puerto de la Laja, El Granado, IV-2001; E. Sánchez Gullón. MA 695314. Idem, Puerto de la Laja, El Granado, verano-2001; E. Sánchez Gullón. MA 681538.

La población que ahora anotamos es la primera cita andaluza de una especie de la que se conocen algunas poblaciones de Galicia y el $\mathrm{N}$ y $\mathrm{C}$ de Portugal (BL, E, R). Ha sido considerada con la categoría "Vulnerable" (VV.AA., loc. cit.2000).

\section{BIBLIOGRAFÍA}

GALLEGO, M. J. -1987-. Scirpus L., in VALDÉS, B., S. TALAVERA ET E. FERNÁNDEZGALIANO (eds.) Flora Vascular de Andalucía Occidental, vol. III. Ketres Editora S.A., Barcelona

GUZMÁN OTANO, D. y D. GOÑI MARTÍNEZ 2004- Eleocharis austriaca Hayek. In VV.AA (eds.) Atlas y Libro Rojo de la Flora Vascular Amenazada de España. Ministerio de Medio Ambiente

LUCEÑO, M. -1994- Monografía del género Carex en la Península Ibérica e islas Baleares. Ruizia 14: pp. 139

LUCEÑO, M. y J. M. MARÍN -2001- Novedades nomenclaturales y corológicas en Ciperáceas ibéricas. Anales Jard. Bot. Madrid 59 (2): 351352

MAIRE, R. -1957-Flore de L'Afrique du Nord, vol. IV

ORTIZ HERRERA, M. A., C. DE VEGA DURÁN, R. BERJANO PÉREZ, E. SÁNCHEZ GULLÓN y S. TALAVERA LOZANO -2004Rhynchospora modesti-lucennoi Castrov., in Bañares et al. (eds.) Atlas y Libro Rojo de la Flora Vascular Amenazada de España. Ministerio de Medio Ambiente

VV.AA. -2000- Lista Roja de la Flora Vascular Española. Conservación Vegetal 6: 39

WALTERS, S. M. -1980- Eleocharis R. Br., in TUTIN, T. G. et al. (eds.) Flora Europaea vol. $V$. Cambridge University Press.

Aceptado para su publicación en octubre de 2006

Dirección de los autores. Área de Botánica. Departamento de Biología Molecular e Ingeniería Bioquímica. Universidad Pablo de Olavide. Ctra de Utrera, km 1 s.n., 41013 Sevilla. 954348383 mlucgar@upo.es; pjimmej@upo.es 